\title{
Association of sex hormone-binding globulin with nonalcoholic fatty liver disease in Chinese adults
}

\author{
Jing Luo ${ }^{1,2+}$, Qian Chen ${ }^{1,2,5 \dagger}$, Tianran Shen ${ }^{1,2}$, Xu Wang $^{1,2}$, Wanjun Fang ${ }^{1,2}$, Xiaocai Wu ${ }^{4}$, Zenan Yuan ${ }^{4}$,
} Gengdong Chen ${ }^{2,3}$, Wenhua Ling ${ }^{1,2^{*}}$ (D) and Yuming Chen ${ }^{2,3^{*}}$

\begin{abstract}
Background: Sex hormone-binding globulin (SHBG), a glycoprotein synthesized by hepatocytes, has been linked to insulin resistance and hepatic lipid metabolism and is suggested to be associated with nonalcoholic fatty liver disease (NAFLD). This study aimed to investigate the association of SHBG with NAFLD in Chinese adults.

Methods: We conducted a community-based, cross-sectional study in China involving 2912 participants aged 4075 years old. All participants underwent detection for hepatic fat infiltration by ultrasound in addition to providing complete medical history and undergoing physical and blood biochemical examinations. The association of serum SHBG with the presence of NAFLD was reported by adjusted odds ratio after applying logistic regression models. To further explore the relationship between SHBG and NAFLD, mRNA expression of SHBG and hepatocyte nuclear factor 4-a (HNF4a), as well as intrahepatic triglycerides, were determined from the liver tissues of 32 subjects with different degrees of steatosis.

Results: Serum SHBG levels in patients with NAFLD (median, $43.8 \mathrm{nmol} / \mathrm{L}$; interquartile range, 33.4-56.8 nmol/L) were significantly lower than those in non-NAFLD subjects (median, $63.4 \mathrm{nmol} / \mathrm{L}$; interquartile range, 47.6-83. $1 \mathrm{nmol} / \mathrm{L}$ ). Serum SHBG levels were inversely correlated with WHR, trunk fat percentage, glucose, HOMA-IR, TG, UA and DHEAS, and were positively correlated with HDL-C levels (all $p<0.001$ ). Logistic regression analysis indicated that serum SHBG levels were negatively associated with the presence of NAFLD in all subjects, as well as the subgroups stratified by sex, BMI and HOMA-IR (all $p<0.05$ ). In human liver tissues, SHBG and HNF4a mRNA expression decreased along with the elevated grade of hepatic steatosis. Both SHBG and HNF4a mRNA expression levels were negatively correlated with intrahepatic triglycerides.
\end{abstract}

Conclusions: These results demonstrate that SHBG levels were negatively associated with the presence of NAFLD in middle-aged and elderly Chinese adults.

Keywords: Sex hormone-binding globulin, Nonalcoholic fatty liver disease, Hepatic steatosis, Intrahepatic triglyceride

\section{Introduction}

Nonalcoholic fatty liver disease (NAFLD) is now acknowledged as a global public health issue [1]. It is estimated that the global prevalence of NAFLD is $25.24 \%$ [2]. In China, $20.09 \%$ of the general adult population is

\footnotetext{
* Correspondence: lingwh@mail.sysu.edu.cn; chenyum@mail.sysu.edu.cn ${ }^{\dagger}$ Jing Luo and Qian Chen contributed equally to this work.

'Department of Nutrition, School of Public Health, Sun Yat-sen University

(Northern Campus), Guangzhou 510080, China

${ }^{2}$ Guangdong Provincial Key Laboratory of Food, Nutrition and Health,

Guangzhou 510080, China

Full list of author information is available at the end of the article
}

potentially influenced by NAFLD [3]. NAFLD has become one of the most common causes of chronic liver disease. It is regarded to be the hepatic manifestation of metabolic syndrome or insulin resistance [4], increasing in parallel with the rise in cardiovascular or cerebrovascular diseases and type 2 diabetes mellitus $[5,6]$. The spectrum of NAFLD ranges from simple liver steatosis to steatohepatitis, which may progress to advanced fibrosis, cirrhosis or hepatocellular carcinoma [7]. Most patients with NAFLD are asymptomatic or complain about nonspecific symptoms (for example, fatigue, right

(c) The Author(s). 2018 Open Access This article is distributed under the terms of the Creative Commons Attribution 4.0 International License (http://creativecommons.org/licenses/by/4.0/), which permits unrestricted use, distribution, and 
upper quadrant discomfort or sleep disorder), which makes it difficult to screen for NAFLD [8].

As our understanding in the occurrence and development of NAFLD continues to evolve, sex hormone-binding globulin (SHBG) has been suggested to be associated with NAFLD. SHBG is a 90-100 KDa homodimeric glycoprotein, mainly secreted by hepatocytes [9]. It has traditionally been considered to function as a transporter of sex steroids, controlling circulating free hormone concentrations [10]. Numerous publications have proposed that low levels of SHBG are strongly associated with the features of metabolic syndrome [11-13] and may play an important role in modifying the risk of insulin resistance and its outcomes [14-16]. An inverse association between SHBG and the risk of NAFLD was observed in diabetic patients and women with polycystic ovarian syndrome [17-20]. Wang $\mathrm{N}$ et al. also demonstrated that lower levels of SHBG were associated with the presence of NAFLD in Chinese men and menopausal women [21]. In contrast to the above findings, the results of a population-based study involving a survey of 1882 men showed that an increase in SHBG levels occurred in men with NAFLD [22]. Though a meta-analysis of associations between testosterone and SHBG and NAFLD which extracted data from 16 observational studies claimed that SHBG was lower in subjects with NAFLD compared to those without NAFLD [23], a significant heterogeneity was observed in the study $\left(\mathrm{I}^{2}=\right.$ $99 \%$ in men, $\mathrm{I}^{2}=97 \%$ in women, both $p$ for heterogeneity $<0.01$ ), which reduced the credibility of the conclusion. Therefore, it is still difficult to draw firm conclusions about the relationship between SHBG and NAFLD for people from the general population, especially when we discuss the problem in the Chinese population.

Nuclear receptor hepatocyte nuclear factor 4- $\alpha$ (HNF4 $\alpha$ ) activates the promoters of multiple genes expressed in hepatocytes that play a role in lipoprotein metabolism [24]. The proximal promoter of the SHBG gene contains an HNF $4 \alpha$ binding site, and overexpression of transcription factor HNF4 $\alpha$ in HepG2 cells can stimulate transcription of the SHBG promoter [25]. Several studies found that monosaccharide and lipid, as well as other factors, could regulate the SHBG expression via changes in HNF4 $\alpha$ gene expression [26-28]. Among them, liver lipids were considered to be a crucial factor in regulating HNF4 $\alpha$-SHBG. Adiponectin treatment of HepG2 cells activated AMPK, which decreased the hepatic lipid content, then increased HNF4 $\alpha$ levels and upregulated SHBG expression [28]. SHBG mRNA is not naturally expressed in rodent liver; therefore, to date, research on SHBG gene expression using in vivo models is limited, and studies concerning SHBG expression in humans are rarely found.

Accordingly, we conducted a large, community-based, cross-sectional study to evaluate the association between
SHBG and the prevalence of NAFLD in middle-aged and elderly Chinese adults. Moreover, we furthered our study by including liver biopsy samples in order to improve our understanding of the relevance between SHBG and the risk of NAFLD in humans.

\section{Methods \\ Participants}

The study was based on the Guangzhou Nutrition and Health Study (GNHS), a community-based prospective cohort study aimed to investigate the potential association between dietary nutrition intake, genetic factors and noncommunicable chronic diseases, along with their interactions. Between July 2008 and June 2010, 3169 participants aged $40-75$ years old who had at least a 5-year continuous residence in Guangzhou were initially recruited into this study through advertisements, health talks, and referrals from local community centres. Of these participants, 2520 completed the initial survey, while 649 subjects dropped out due to refusal, loss of contact, emigration or death between April 2011 and May 2013. Additional 869 participants were recruited between March 2013 and October 2013 using the same methods. Information on demographic characteristics were collected in both 2008-2010 and 2011-2013. Fasting venous blood samples were obtained and B-scan ultrasonography was measured during the 2011-2013 survey.

Participants who had missing blood biochemical index values or were without abdominal ultrasonographic results were excluded, and 3006 participants remained available for analysis. Furthermore, subjects with any of the following conditions were excluded: excessive alcohol consumption ( $\geq 140 \mathrm{~g} / \mathrm{wk}$. for males or $\geq 70 \mathrm{~g} / \mathrm{wk}$. for females) [29]; viral hepatitis [29]; other causes of liver disease (autoimmune, genetic, etc.); haemochromatosis [30]; biliary obstructive diseases; the presence of severe medical diseases (cancer, stroke, heart failure, etc.); current treatment with systemic corticosteroids; and pregnancy [30]. In the end, 2912 participants were enrolled in this analysis (the flow-chart of the study is presented in Fig. 1).

\section{Data collection}

Each participant underwent a comprehensive physical examination, routine biochemical analysis of fasting blood, hepatitis virus test and B-scan ultrasonography. Trained researchers administered face-to-face interviews to collect information on participants' demographic characteristics, lifestyle and habits (e.g., smoking, alcohol drinking and physical activity) and history of chronic diseases through standardized questionnaires. Subjects who drank alcohol once a week or smoked at least one cigarette per day for at least 6 months were identified as 


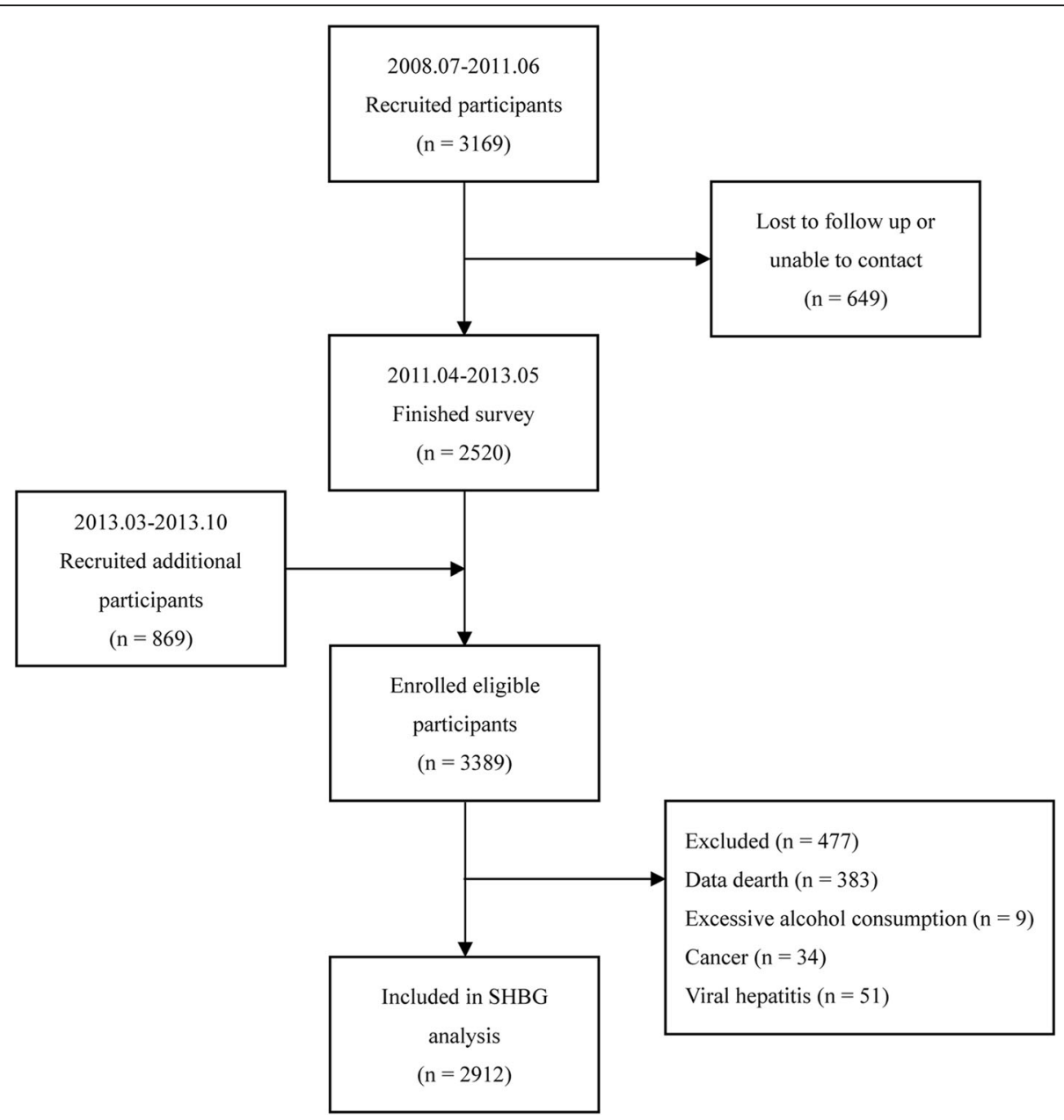

Fig. 1 Flow-chart of the recruitment of community participants

drinkers or smokers. Body mass index (BMI) was calculated as weight $(\mathrm{kg})$ divided by the square of height $\left(\mathrm{m}^{2}\right)$, and subjects with values greater than $24 \mathrm{~kg} / \mathrm{m}^{2}$ were identified as overweight by the Chinese standard proposed by the Working Group on Obesity in China [31]. The metabolic equivalent (MET) intensity was calculated to estimate daily physical activity using a 24-h physical activity questionnaire [32]. The fat mass of the trunk region was quantified using dual-energy $\mathrm{X}$-ray absorptiometry scans (Discovery W; Hologic Inc., Waltham, MA, USA). The trunk region was defined as the area of the trunk between an upper horizontal boundary below the chin and a lower boundary above the oblique lines through each hip joint [33].

\section{Clinical diagnosis and rating for NAFLD}

Abdominal ultrasonography was used to diagnose NAFLD on a Doppler sonography machine (Sonoscape SSI-5500, Shenzhen, China) with a $3.5 \mathrm{MHz}$ probe. Ultrasound scanning for all participants was performed by two experienced technicians in the same clinical setting who were blinded to the clinical and laboratory data. The diagnosis of NAFLD was conducted following standard criteria issued by the Fatty Liver Disease Study Group of the Chinese Liver Disease Association [29, 34], and hepatic steatosis was semi-quantitatively rated as absent, mild, moderate or severe on the basis of ultrasonographic phenomenon including hepatorenal echo contrast, liver brightness, deep attenuation and vessel structure blurring. Between-operator reliability evaluation for ultrasound scanning was determined in 100 participants, showing a very good precision (Spearman's $r=0.911$, kappa $=0.875$, total agreement $=93 \%, p<0.001)$. Validity evaluation was assessed in 34 participants who further underwent computed tomography $(\mathrm{CT})$, with the technicians blinded to the ultrasound results, a good agreement was observed (Spearman's $r=0.905$, kappa $=0.691$, total agreement $=85 \%, p<0.001$ ).

\section{Human liver tissue}

Thirty-two patients undergoing hepatic resection as treatment for hepatic benign tumour in The Third Affiliated 
Hospital of Sun Yat-sen University were recruited for this study, which was approved by Sun Yat-sen University's School of Public Health Ethics Committee. Following informed consent, the patients' medical history was reviewed, and biochemical parameters were collected. Subjects with excessive alcohol consumption and other liver diseases, such as hepatitis B, hepatitis $\mathrm{C}$ and cirrhosis were excluded. After surgery to remove a liver specimen, tumour was removed for clinical analysis, and adjacent normal liver was stored in liquid nitrogen for subsequent analysis.

The degree of steatosis in sections of the liver specimens were assessed by two pathologists in a blinded fashion. The histological features of the liver were graded according to a histological scoring system for NAFLD [35].

\section{Biochemical measurements}

All venous blood samples were obtained after overnight fasting. The serum was separated into several aliquots and stored at $-80{ }^{\circ} \mathrm{C}$ within $2 \mathrm{~h}$. Serum glucose, triglycerides (TG), total cholesterol (TC), high-density lipoprotein cholesterol (HDL-C), low-density lipoprotein cholesterol (LDL-C), aspartate aminotransferase (AST), alanine aminotransferase (ALT) and uric acid (UA) were determined enzymatically on a Hitachi 7600-010 automated analyser (Hitachi, Tokyo, Japan). Insulin resistance was evaluated using the homeostasis model assessment (HOMA) index [fasting glucose $(\mathrm{mmol} / \mathrm{L}) \times$ insulin $(\mu \mathrm{U} / \mathrm{mL}) / 22.5$. Serum SHBG, testosterone (Testo) and dehydroepiandrosterone sulphate (DHEAS) were measured by chemiluminescent enzyme immunometric assay (CLIA) using kits obtained from Abbott Corporation (Chicago, IL, USA) with an Abbott ARCHITECT I2000 automated analyser. The minimum detectable dose of SHBG was $2 \mathrm{nmol} / \mathrm{L}$, and the intra- and inter-assay coefficients of variation for SHBG were $4.1 \%$ and $5.8 \%$, respectively.

\section{RNA extraction and quantitative real-time PCR}

Total RNA from human liver tissues was isolated using TRIzol reagent (Invitrogen, Carlsbad, CA, USA), and reverse transcription was performed with a PrimeScript RT Reagent Kit (TaKaRa, Tokyo, Japan) according to the manufacturer's instructions. The mRNA expression was quantified in a 96-well plate using a 7500 Real-Time PCR System (Applied Biosystems, Inc., Foster City, CA, USA) with SYBR Green Master Mix (TaKaRa, Tokyo, Japan). The relative mRNA expression levels were determined using the $2^{-\Delta \Delta C t}$ method with GAPDH as an internal control for normalization. The sequences of forward and reverse primers used in the current study were: human SHBG, 5'-GCCCAGGACAAGAGCCTATC-3' and 5'-CC TTAGGGTTGGTATCCCCATAA-3'; human HNF4 $\alpha$, 5'AACACCTCAACAAGGGCACTC-3' and 5'-CCCCACT TGAAACG-GTTCCT-3'; human GAPDH, 5'-GGAGCG
AGATCCCTCCAAAAT-3' and 5'-GGCTGTTGTCATAC TTCTCATGG -3'.

\section{Analysis of liver TG content}

Human liver samples were immediately shock-frozen and grinded in liquid nitrogen to measure the intrahepatic TG concentration, which was determined enzymatically after extraction using a commercial kit (Applygen Technologies Inc., Beijing, China) according to the manufacturer's instructions.

\section{Statistical analysis}

Data were presented as means \pm standard deviation or median (interquartile range) for continuous variables and as frequencies (percentages) for categorical variables. Differences between groups of continuous variables were compared using either independent samples t-test or Mann-Whitney U test. Chi-square test was used to compare categorical variables between groups. Spearman's correlation analysis and multiple linear regression were used to estimate the association between serum SHBG levels and several relevant metabolic factors. Logistic regression analysis was used to estimate the odds ratios (ORs) and 95\% confidence intervals (CIs) for the risk of NAFLD with increasing quartiles of serum SHBG levels, using the lowest quartile as the reference group. Logistic regression analyses were performed separately for different sexes, different BMI and HOMA-IR status, and the interactions between serum SHBG concentrations and BMI levels as well as HOMA-IR were tested. We adjusted for age, sex, postmenopausal status (for females) and household income in Model 1. To investigate the independent associations, we further adjusted for waist-to-hip ratio (WHR), trunk fat percentage, current smoking and drinking, physical activity (MET), hypertension and diabetes in Model 2, plus serum glucose, HOMA-IR, ALT, triglycerides, HDL-C, UA, testosterone and DHEAS levels in Model 3. Analysis of covariance (ANCOVA) was used to identify differences between groups, and the variables were adjusted as in Model 3. Kruskal-Wallis test was used as appropriate for comparisons of mRNA expression in liver tissue between groups, and multiple testing was corrected using Bonferroni correction. Correlations of hepatic TG with serum SHBG levels, SHBG mRNA expression and $H N F 4 \alpha$ mRNA expression in liver tissue were examined using Pearson's correlation analysis. A two-tailed $p$-value $<0.05$ was considered statistically significant. All of the statistical procedures were performed using SPSS Statistics software (version 22.0, SPSS Inc., Chicago, IL, USA).

\section{Results}

\section{Characteristics of participants}

The characteristics of the participants are presented in Additional file 1: Table S1. The study involved 2912 
participants, of whom 2009 were women. NAFLD group presented with higher BMI levels than non-NAFLD group, as well as a higher WHR and trunk fat percentage. In addition, participants with NAFLD had higher serum levels of ALT, glucose, HOMA-IR, TG and UA, and lower physical activity and HDL-C levels than non-NAFLD subjects in both females and males (all $p<0.05$ ).

\section{Serum SHBG levels in non-NAFLD and NAFLD subjects}

In this study, the non-NAFLD participants had much higher SHBG concentrations (median, $63.4 \mathrm{nmol} / \mathrm{L}$; interquartile range, $47.6-83.1 \mathrm{nmol} / \mathrm{L}$ ) than the NAFLD participants (median, $43.8 \mathrm{nmol} / \mathrm{L}$; interquartile range, 33.4-56.8 nmol/L) $(p<0.001)$. Additionally, as shown in Fig. 2a-c, serum SHBG concentrations exhibited significant differences between non-NAFLD subjects and NAFLD patients regardless of sex (median, $67.9 \mathrm{nmol} / \mathrm{L}$ versus $45.6 \mathrm{nmol} / \mathrm{L}$ in females; $54.8 \mathrm{nmol} / \mathrm{L}$ versus 40.6 $\mathrm{nmol} / \mathrm{L}$ in males), BMI (median, $67.6 \mathrm{nmol} / \mathrm{L}$ versus $49.0 \mathrm{nmol} / \mathrm{L}$ in subjects with $\mathrm{BMI}<24 \mathrm{~kg} / \mathrm{m}^{2} ; 51.7 \mathrm{nmol} /$ $\mathrm{L}$ versus $40.2 \mathrm{nmol} / \mathrm{L}$ in subjects with $\mathrm{BMI} \geq 24 \mathrm{~kg} / \mathrm{m}^{2}$ ) and HOMA-IR status (median, $66.3 \mathrm{nmol} / \mathrm{L}$ versus
$49.7 \mathrm{nmol} / \mathrm{L}$ in subjects with HOMA-IR $<2 ; 52.3 \mathrm{nmol} / \mathrm{L}$ versus $38.8 \mathrm{nmol} / \mathrm{L}$ in subjects with $\mathrm{HOMA}-\mathrm{IR} \geq 2$ ) (all $p$ $<0.001)$.

As shown in Fig. 2d, we observed that serum SHBG levels (log-transformed) decreased gradually with increased severity of hepatic steatosis in both females and males after adjustments for the variables [age, postmenopausal status (for females), household income, WHR, trunk fat, current smoking and drinking, physical activity, hypertension and diabetes, serum glucose, insulin resistance, TG, HDL-C, ALT, UA, testosterone and DHEAS levels] (both $p$-trend $<0.05$ ).

\section{Association between serum SHBG levels and metabolic risk factors}

We next investigated the correlation of serum SHBG levels with a cluster of anthropometric parameters and biochemical indices. The results are shown in Table 1. The analysis demonstrated negative correlations of serum SHBG with sex, BMI, WHR, trunk fat percentage and several biochemical parameters, including ALT, glucose, HOMA-IR, TG, TC, UA, testosterone and DHEAS

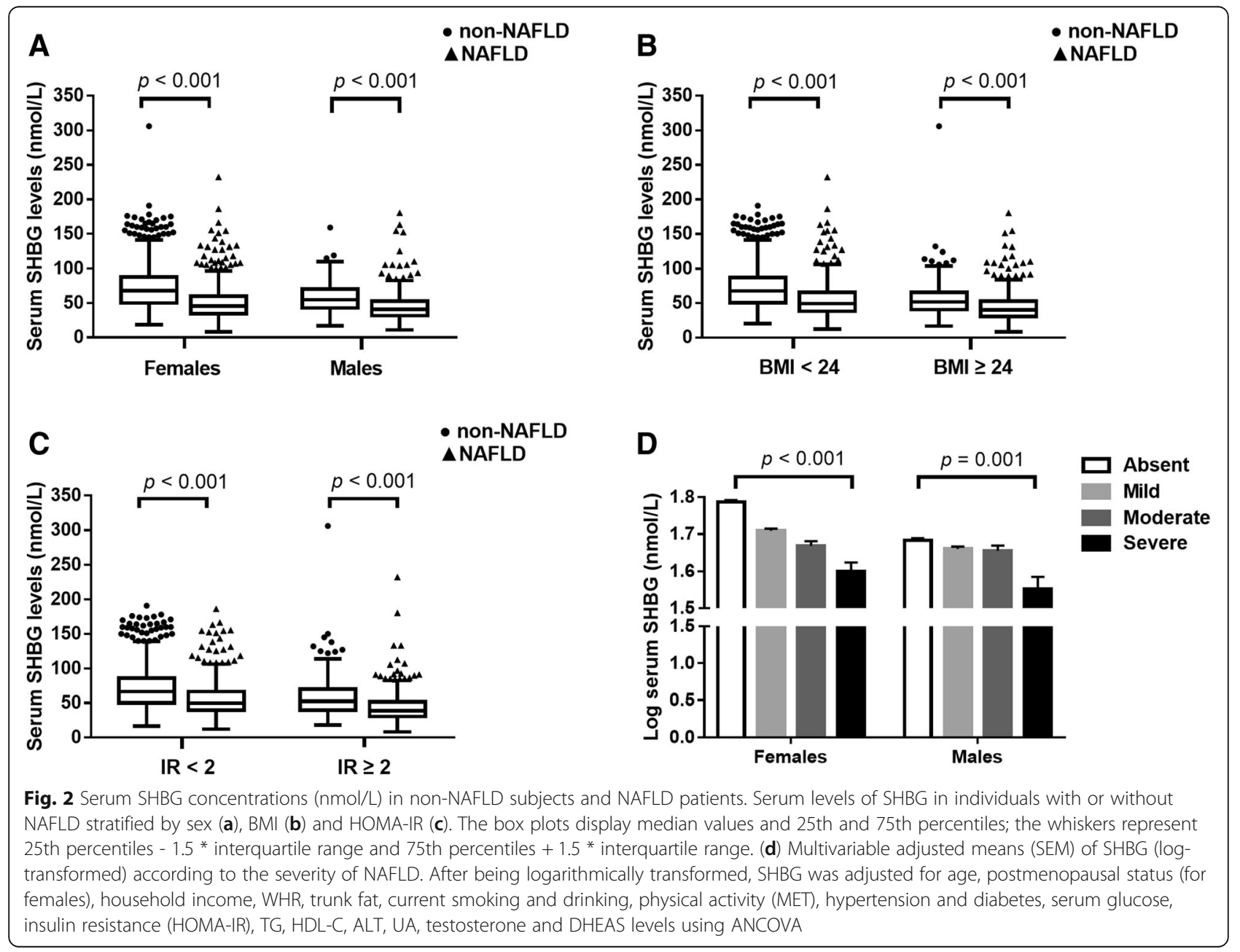


(all $p<0.001$ ). A positive correlation of SHBG with HDL-C levels was observed $(p<0.001)$. After adjustment for age, sex and BMI, correlation of serum SHBG levels with WHR, trunk fat percentage, glucose, HOMA-IR, TG, UA, DHEAS and HDL-C still existed robustly (all $p<0.001$ ). We further conducted multiple regression analysis to examine the relationships that involved the above parameters. As shown in Additional file 2: Table S2, serum SHBG levels (log-transformed) were treated as a dependent variable. In all subjects, BMI, WHR, trunk fat, ALT, fasting glucose, HOMA-IR, TG, HDL-C, cholesterol, LDL-C and UA were all chosen as independent variables before and after adjusting for age, sex, current smoking and drinking, physical activity, hypertension, diabetes (except for cholesterol and LDL-C, all $p<0.001$ ).

\section{Association of serum SHBG levels with NAFLD}

To further explore the associations of serum SHBG levels with NAFLD, we employed logistic regression models to examine the sex-specific associations of serum SHBG quartiles with the odds of the presence of NAFLD. As shown in Table 2, We found that higher serum SHBG quartiles were associated with lower odds of NAFLD in all subjects (OR for the highest quartile versus the lowest quartile, $0.09 ; 95 \% \mathrm{CI}, 0.07-0.11 ; p<0.001$ ) after adjusting for age, sex and household income in binary logistic regression. In the following analysis stratified by sex, the initial association remained the same in both females and males (all $p<0.001$ ). Furthermore, these associations still maintained robustness after adjustment for physical and metabolic parameters in Models 2 and 3, respectively. The adjusted ORs of NAFLD in Model 3 for the highest (versus the lowest) quartile were 0.24 (95\% CI, 0.18-0.32) in all participants, 0.19 (95\% CI, 0.13-0.27) in females and 0.42 (95\% CI, 0.23-0.77) in males (all $p<0.05$ ). Otherwise, we still found significant inverse associations between SHBG and NAFLD regardless of BMI ( $p$ for interaction $=0.150$; for subjects with $\mathrm{BMI}<24 \mathrm{~kg} / \mathrm{m}^{2}$, adjusted OR in Model 3, 0.31, 95\% CI, 0.21-0.45, $p$ $<0.001$; for subjects with BMI $\geq 24 \mathrm{~kg} / \mathrm{m}^{2}$, adjusted OR in Model 3, 0.31, 95\% CI, 0.19-0.51, $p<0.001$ ) and HOMA-IR status ( $p$ for interaction $=0.021$; for subjects with HOMA-IR $<2$, adjusted OR in Model 3, 0.34, 95\% CI, 0.24-0.49, $p<0.001$; for subjects with HOMA-IR $\geq$ 2, adjusted OR in Model 3, 0.22, 95\% CI, 0.13-0.36, $p<0.001$ ) (Table 3).

\section{SHBG expression in liver tissue of NAFLD patients}

We next examined the expression of SHBG mRNA in liver tissue obtained from 32 patients. The characteristics of the participants are presented in Additional file 2: Table S3. A positive correlation was found between the expression of SHBG mRNA in liver and the level of serum SHBG $(r=0.788, p<0.001$; Fig. 3a). The liver SHBG mRNA expression and serum SHBG level of Grade 1 steatosis was respectively 2.78 - and 1.43-fold lower than that in tissues of Grade $0(p<0.05)$. Due to the small sample sizes in Grades 2 and 3, the two groups were combined for analysis. In Grade 2-3 liver tissues,

Table 1 Univariate and multivariate-adjusted Spearman correlation coefficients of SHBG and metabolic risk factors

\begin{tabular}{|c|c|c|c|c|}
\hline \multirow[t]{2}{*}{ Variables } & \multicolumn{2}{|l|}{ SHBG } & \multicolumn{2}{|c|}{ SHBG (age, sex and BMI-adjusted) } \\
\hline & $\bar{r}$ & $p$ & $r$ & $p$ \\
\hline Age (years) & 0.027 & 0.144 & - & - \\
\hline Sex & -0.177 & $<0.001$ & - & - \\
\hline $\mathrm{BMI}\left(\mathrm{kg} / \mathrm{m}^{2}\right)$ & -0.455 & $<0.001$ & - & - \\
\hline WHR & -0.327 & $<0.001$ & -0.186 & $<0.001$ \\
\hline Trunk fat percentage (\%) & -0.205 & $<0.001$ & -0.232 & $<0.001$ \\
\hline $\operatorname{ALT}(U / L)$ & -0.206 & $<0.001$ & -0.029 & 0.117 \\
\hline Glucose (mmol/L) & -0.208 & $<0.001$ & -0.114 & $<0.001$ \\
\hline HOMA-IR & -0.467 & $<0.001$ & -0.177 & $<0.001$ \\
\hline Triglyceride (mmol/L) & -0.386 & $<0.001$ & -0.192 & $<0.001$ \\
\hline Cholesterol (mmol/L) & -0.067 & $<0.001$ & -0.015 & 0.406 \\
\hline $\mathrm{HDL}-\mathrm{C}(\mathrm{mmol} / \mathrm{L})$ & 0.390 & $<0.001$ & 0.269 & $<0.001$ \\
\hline LDL-C (mmol/L) & 0.023 & 0.215 & -0.026 & 0.168 \\
\hline UA ( $\mu \mathrm{mol} / \mathrm{L})$ & -0.331 & $<0.001$ & -0.206 & $<0.001$ \\
\hline Testosterone (ng/dL) & -0.105 & $<0.001$ & 0.235 & $<0.001$ \\
\hline DHEAS ( $\mu \mathrm{g} / \mathrm{dL})$ & -0.216 & $<0.001$ & -0.122 & $<0.001$ \\
\hline
\end{tabular}

The age, sex and BMI-adjusted associations between serum SHBG levels and several relevant factors were estimated by partial correlation SHBG sex hormone-binding globulin; BMI body mass index; WHR waist-to-hip ratio; $A L T$ alanine aminotransferase; HOMA-IR homeostasis model assessment of insulin resistance; $H D L-C$ high-density lipoprotein cholesterol; $L D L-C$ low-density lipoprotein cholesterol; UA uric acid; $D H E A S$ dehydroepiandrosterone sulphate 
Table 2 Adjusted odds ratios (95\% confidence intervals) of NAFLD according to sex and serum SHBG quartiles using adjusted logistic regression

\begin{tabular}{|c|c|c|c|c|c|}
\hline & \multicolumn{4}{|c|}{ SHBG (nmol/L) } & \multirow[t]{2}{*}{$p$ for tren } \\
\hline & Quartile 1 & Quartile 2 & Quartile 3 & Quartile 4 & \\
\hline Total $(N=2912)$ & $<38.70$ & $38.70-52.45$ & $52.46-71.3$ & $>71.3$ & \\
\hline Model 1 & 1.00 & $0.38(0.30-0.48)$ & $0.21(0.16-0.26)$ & $0.09(0.07-0.11)$ & $<0.001$ \\
\hline Model 2 & 1.00 & $0.46(0.36-0.59)$ & $0.30(0.23-0.38)$ & $0.18(0.13-0.23)$ & $<0.001$ \\
\hline Model 3 & 1.00 & $0.54(0.42-0.70)$ & $0.38(0.29-0.50)$ & $0.24(0.18-0.32)$ & $<0.001$ \\
\hline Females $(N=2009)$ & $<40.65$ & $40.65-55.70$ & $55.71-75.80$ & $>75.80$ & \\
\hline Model 1 & 1.00 & $0.34(0.25-0.45)$ & $0.18(0.14-0.24)$ & $0.07(0.05-0.09)$ & $<0.001$ \\
\hline Model 2 & 1.00 & $0.40(0.30-0.54)$ & $0.24(0.18-0.33)$ & $0.13(0.09-0.18)$ & $<0.001$ \\
\hline Model 3 & 1.00 & $0.49(0.36-0.67)$ & $0.33(0.24-0.45)$ & $0.19(0.13-0.27)$ & $<0.001$ \\
\hline Males $(N=903)$ & $<35.50$ & $35.50-47.10$ & $47.11-61.00$ & $>61.00$ & \\
\hline Model 1 & 1.00 & $0.67(0.45-1.00)$ & $0.31(0.21-0.46)$ & $0.13(0.09-0.21)$ & $<0.001$ \\
\hline Model 2 & 1.00 & $0.83(0.53-1.29)$ & $0.47(0.30-0.73)$ & $0.35(0.22-0.57)$ & $<0.001$ \\
\hline Model 3 & 1.00 & $0.95(0.59-1.53)$ & $0.57(0.35-0.95)$ & $0.42(0.23-0.77)$ & 0.001 \\
\hline
\end{tabular}

Model 1: Adjusted for age, sex, postmenopausal status (for females) and household income

Model 2: Adjusted for the variables in Model 1 plus WHR, trunk fat, current smoking and drinking, physical activity (MET), hypertension and diabetes Model 3: Adjusted for the variables in Model 2 plus serum glucose, insulin resistance (HOMA-IR), TG, HDL-C, ALT, UA, testosterone and DHEAS levels NAFLD, nonalcoholic fatty liver disease; SHBG, sex hormone-binding globulin

Table 3 Adjusted odds ratios (95\% confidence intervals) of NAFLD according to BMI and HOMA-IR status and serum SHBG quartiles using adjusted logistic regression

\begin{tabular}{|c|c|c|c|c|c|}
\hline & \multicolumn{4}{|c|}{ SHBG (nmol/L) } & \multirow[t]{2}{*}{$p$ for trend } \\
\hline & Quartile 1 & Quartile 2 & Quartile 3 & Quartile 4 & \\
\hline $\begin{array}{l}\mathrm{BMI}<24 \mathrm{~kg} / \mathrm{m}^{2} \\
(\mathrm{~N}=1696)\end{array}$ & $<45.03$ & $45.03-60.90$ & $60.91-80.98$ & $>80.98$ & \\
\hline Model 1 & 1.00 & $0.50(0.38-0.66)$ & $0.23(0.17-0.31)$ & $0.15(0.11-0.21)$ & $<0.001$ \\
\hline Model 2 & 1.00 & $0.56(0.42-0.75)$ & $0.29(0.21-0.40)$ & $0.24(0.17-0.34)$ & $<0.001$ \\
\hline Model 3 & 1.00 & $0.60(0.46-0.84)$ & $0.35(0.25-0.48)$ & $0.31(0.21-0.45)$ & $<0.001$ \\
\hline $\begin{array}{l}\mathrm{BMI} \geq 24 \mathrm{~kg} / \mathrm{m}^{2} \\
(\mathrm{~N}=1216)\end{array}$ & $<32.70$ & $32.70-43.10$ & $43.11-56.30$ & $>56.30$ & \\
\hline Model 1 & 1.00 & $0.53(0.34-0.83)$ & $0.33(0.21-0.51)$ & $0.17(0.11-0.27)$ & $<0.001$ \\
\hline Model 2 & 1.00 & $0.57(0.36-0.89)$ & $0.37(0.24-0.57)$ & $0.21(0.14-0.32)$ & $<0.001$ \\
\hline Model 3 & 1.00 & $0.70(0.44-1.12)$ & $0.49(0.30-0.78)$ & $0.31(0.19-0.51)$ & $<0.001$ \\
\hline$p$ for interaction & - & - & - & - & 0.150 \\
\hline $\begin{array}{l}\text { HOMA-IR }<2 \\
(N=1780)\end{array}$ & $<45.20$ & $45.20-60.10$ & $60.11-79.38$ & $>79.38$ & \\
\hline Model 1 & 1.00 & $0.55(0.42-0.72)$ & $0.29(0.22-0.39)$ & $0.17(0.13-0.23)$ & $<0.001$ \\
\hline Model 2 & 1.00 & $0.64(0.49-0.85)$ & $0.40(0.29-0.54)$ & $0.32(0.23-0.44)$ & $<0.001$ \\
\hline Model 3 & 1.00 & $0.67(0.50-0.89)$ & $0.43(0.31-0.59)$ & $0.34(0.24-0.49)$ & $<0.001$ \\
\hline $\begin{array}{l}\text { HOMA-IR } \geq 2 \\
(N=1132)\end{array}$ & $<31.50$ & $31.50-41.60$ & $41.61-55.68$ & $>55.68$ & \\
\hline Model 1 & 1.00 & $0.76(0.47-1.21)$ & $0.41(0.27-0.64)$ & $0.16(0.10-0.25)$ & $<0.001$ \\
\hline Model 2 & 1.00 & $0.84(0.52-1.36)$ & $0.46(0.29-0.73)$ & $0.21(0.14-0.33)$ & $<0.001$ \\
\hline Model 3 & 1.00 & $0.83(0.50-1.36)$ & $0.43(0.27-0.70)$ & $0.22(0.13-0.36)$ & $<0.001$ \\
\hline$p$ for interaction & - & - & - & - & 0.021 \\
\hline
\end{tabular}

Model 1: Adjusted for age, sex and household income

Model 2: Adjusted for the variables in Model 1 plus WHR, trunk fat, current smoking and drinking, physical activity (MET), hypertension and diabetes Model 3: Adjusted for the variables in Model 2 plus serum glucose, insulin resistance (HOMA-IR), TG, HDL-C, ALT, UA, testosterone and DHEAS levels NAFLD nonalcoholic fatty liver disease; SHBG sex hormone-binding globulin; BMI body mass index; HOMA-IR homeostasis model assessment of insulin resistance 


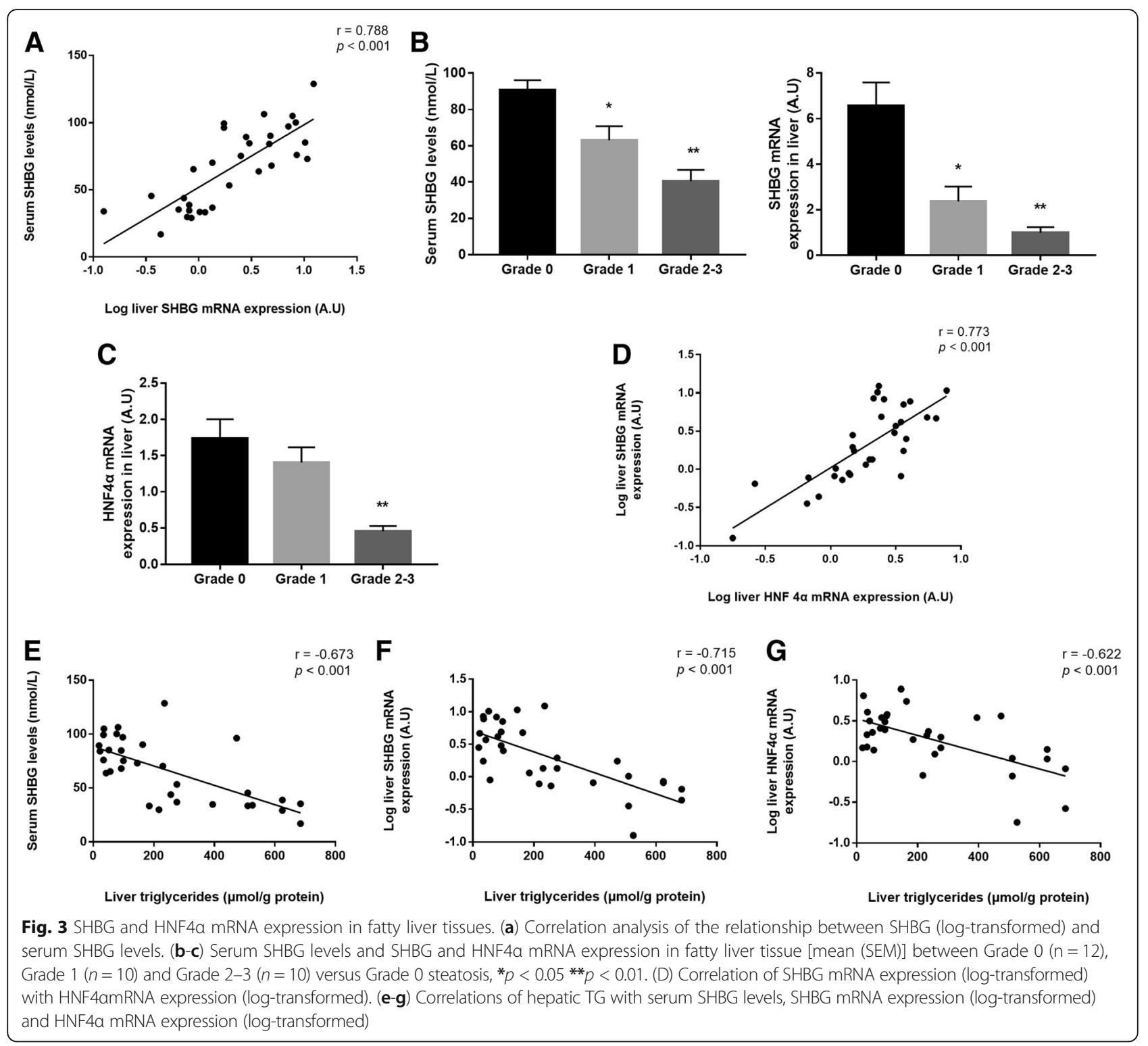

the SHBG mRNA expression and serum SHBG level was respectively 6.72- and 2.23-fold lower than that in tissues of Grade $0(p<0.001)$ (Fig. 3b). As shown in Fig. 3c, HNF4 $\alpha$ mRNA expression in Grade 2-3 was 3.82-fold lower than that in Grade $0(p<0.001)$. SHBG mRNA expression levels were positively correlated with HNF4 $\alpha$ mRNA $(r=0.773, p<0.001$; Fig. 3d). Further results revealed that intrahepatic TG concentrations had significantly negative correlations with SHBG $(r=$ $-0.715, p<0.001)$ and HNF4 $\alpha(r=-0.622, p<0.001)$ mRNA expression levels in liver, as well as serum SHBG levels $(r=-0.673, p<0.001)$ (Fig. 3e-g).

\section{Discussion}

The results of our community-based study illustrated an inverse association between serum levels of SHBG and the prevalence of NAFLD. In this study, the NAFLD participants had much lower SHBG concentrations than the non-NAFLD participants, and serum SHBG levels tended to decrease significantly with the increase of NAFLD severity in both females and males. Furthermore, we found that hepatic SHBG mRNA expression in 32 liver biopsy samples decreased as the grade of hepatic steatosis elevated. Our data also suggested that both serum SHBG levels and liver SHBG and HNF4 $\alpha$ expression were negatively correlated with hepatic triglycerides.

Past research on the relationships between SHBG and NAFLD has often been performed in patients with metabolic disorders [11-16], and the results showed that serum SHBG levels were lower in NAFLD patients with metabolic disorders than in those without NAFLD. However, in these patients, the SHBG levels were lower 
than normal, so it is hard to apply these results to healthy populations. Additionally, due to the small sample sizes of these studies, the results have been inconclusive $[12,17,18]$. The present results that low SHBG groups were associated with higher odds of NAFLD in middle-aged and elderly Chinese adults are in line with those from a previous study reported by Wang $\mathrm{N}$ et al. [21]. However, the prevalence of diabetes, serum TG and BMI levels in their study were all much higher than that in our community population. Furthermore, the authors did not perform the subgroup analysis to exclude metabolic disorders, therefore limiting the conclusions from their study. Our study was based on a large, communitybased, middle-aged and elderly Chinese population, after multivariate fully adjusted, participants in the highest quartile of SHBG compared with those in the lowest quartile of SHBG had a significantly lower prevalence of NAFLD (OR, 0.19, 95\% CI, 0.13-0.27 in females; OR, 0.42 , 95\% CI, $0.23-0.77$ in males; both $p<0.05$ ). Research has shown a correlation between NAFLD and $\mathrm{BMI}$, as the prevalence of NAFLD was reportedly lower in individuals with normal BMI levels and without metabolic risk factors [36]. NAFLD was also shown to have an obvious association with insulin resistance, which is a key risk factor for the development of type 2 diabetes mellitus [37]. Therefore, to estimate the association between SHBG and the risk of NAFLD without the confounding influences of overweight and insulin resistance, we further performed the subgroup analysis after stratifying the whole population by BMI and HOMA-IR status. Inverse associations between SHBG and the presence of NAFLD were still observed regardless of BMI and HOMA-IR status.

Two potential reasons may explain the association between SHBG and NAFLD. First, the occurrence and progression of the NAFLD may downregulate the hepatic expression of SHBG. Previous studies reported that both the accumulation of hepatic lipids [28] and chronic inflammation (pathogenesis of NAFLD) [38-40] could decrease the expression of SHBG. Other metabolic disorders concomitant with NAFLD, such as hyperglycaemia might also directly decrease the expression of SHBG [41]. Second, SHBG itself might contribute to the development of metabolic disorders. Ding EL et al. found that low circulating levels of SHBG played a strong causal role in the occurrence of type 2 diabetes in mendelian randomization analyses [42]. Moreover, SHBG overexpression in C57BL/ $\mathrm{ksJ-db/db}$ mice reduced body weight gain [43], and SHBG overexpression in mouse models of NAFLD could significantly reduce liver fat accumulation by reducing key lipogenic enzymes [44].

As we know, liver tissue biopsy is the gold standard for diagnosing NAFLD and determining the grade of steatosis. To the best of our knowledge, our study was the first to investigate the relationship between SHBG and NAFLD in Asian biopsy-proven patients, although not in a large sample size. Our results revealed that HNF4 $\alpha$ and SHBG mRNAs were significantly lower in subjects with high grades of hepatic steatosis, in accordance with a significantly decreasing trend in serum SHBG levels along with the elevated severity in our NAFLD subjects diagnosed by ultrasonography. Moreover, hepatic expression of HNF4 $\alpha$ mRNA was positively correlated with SHBG mRNA. There were significantly inverse linear relationships between hepatic triglyceride concentrations and the levels of both SHBG and HNF4a mRNA expression levels. Serum SHBG levels were lower in subjects with high concentrations of hepatic triglycerides. Our results reveal a potential mechanism in that the accumulation of lipid in human liver is an important influencing factor of SHBG gene expression, thereby circulating serum levels of SHBG, and these effects are perhaps mediated through changes in nuclear transcription factor HNF4 $\alpha$. These results further supported the findings in our community-based study that serum SHBG is associated with NAFLD risk. Additionally, as the concentration of serum SHBG significantly decreased with the elevated degree of liver steatosis and injury, it can potentially be used to monitor the clinical response to interventions in the management of patients with NAFLD.

The prevalence of NAFLD in this middle-aged and elderly population, as diagnosed by ultrasound, was estimated to be $50.8 \%$. We hypothesize that the following reasons can account for the high prevalence. First, the prevalence of NAFLD increases with age [45], and the average age of the participants in our community-based study was 60.6 years. Second, there may be selection bias in this research, as people with underlying health problems were more willing to participate in our study. Third, although the overall pooled prevalence of NAFLD was $20.09 \%$ in China [3], the epidemiology of NAFLD is greatly shaped by tremendous differences in lifestyle and economic growth between urban and rural areas within the same country. The relatively high prevalence of NAFLD in urbanized Asian areas including China has been reported [46-48], which was consistent with our results.

There were several limitations of this study, including a relatively limited biopsy-proven sample size. The diagnosis of NAFLD was based on ultrasonography, which is non-invasive, low cost, accessible, and widely used in large epidemiological studies, despite not being the gold standard as liver biopsy. Female subjects in the present study were more than double the proportion of males, and in order to avoid the potential bias, we conducted subgroup analysis after stratifying the whole population by sex. The results of logistic regression analysis showed 
that the associations between SHBG and the risk of NAFLD were consistent in females and males. Finally, this was a cross-sectional study design, which precluded us from establishing causal relationship. Further prospective studies are needed to evaluate the temporal relationship between SHBG and hepatic damage or predict how changes in SHBG will affect the progression of NAFLD.

\section{Conclusions}

In summary, we found an inverse association between serum SHBG levels and the presence of NAFLD after multivariable adjustment for metabolic risk factors in community-dwelling participants. Research on liver specimens showed that not only did serum concentrations of SHBG decrease as the degree of hepatic steatosis exacerbated in NAFLD patients but also the liver mRNA expression of SHBG was negatively correlated with the severity of liver injury and intrahepatic TG. Whether SHBG can be selected as a noninvasive biomarker for NAFLD will be determined in future prospective studies and clinical trials.

\section{Additional files}

Additional file 1: Table S1. Demographic characteristics, physical and metabolic measurements by sex and NAFLD status. (DOC 72 kb)

Additional file 2: Table S2. Association of serum SHBG levels with metabolic risk factors using multiple linear regression analysis. Table S3. Anthropometric and clinical parameters of control subjects and patients with NAFLD. (DOC 50 kb)

\section{Abbreviations}

ALT: Alanine aminotransferase; ANCOVA: Analysis of covariance; AST: Aspartate aminotransferase; BMI: Body mass index; Cl: Confidence interval; DHEAS: Dehydroepiandrosterone sulphate; HDL-C: High-density lipoprotein cholesterol; HNF4a: Hepatocyte nuclear factor 4-a; HOMA-IR: Homeostasis model assessment of insulin resistance; LDL-C: Low-density lipoprotein cholesterol; MET: Metabolic equivalent; NAFLD: Nonalcoholic fatty liver disease; OR: Odds ratio; SHBG: Sex hormone-binding globulin; TC: Total cholesterol; Testo: Testosterone; TG: Triglycerides; UA: Uric acid; WHR: Waist-to-hip ratio

\section{Acknowledgements}

We thank all volunteers for their participation.

\section{Funding}

This research was supported by grants from the Guangzhou science and technology project (42050027)

\section{Availability of data and materials}

The datasets generated and/or analysed during the current study are not publicly available, since ethics approval and participants' consent does not allow public sharing of data, but are available from the corresponding author on reasonable request.

\section{Authors' contributions}

$J L, Q C, Y M C a n d$ WHL developed the overall research plan and had study oversight; JL, QC, TRS, XW conducted the whole research; WJF, XCW, ZNY and GDC participated in the collection of the data and samples of population study; JL and QC analyzed the data; JL and QC wrote the manuscript and had the primary responsibility for the final content. All authors read and approved the final manuscript.

\section{Ethics approval and consent to participate}

Written informed consent was provided by all participants, and the study was approved by the Ethics Committee of the School of Public Health at Sun Yat-sen University (Approval Number ZDGWYL2009-3, Registration Number NCT03179657, www.ClinicalTrials.gov), which meets the ethical standards established in the Declaration of Helsinki.

\section{Consent for publication}

Not applicable.

\section{Competing interests}

The authors declare that they have no competing interests.

\section{Publisher's Note}

Springer Nature remains neutral with regard to jurisdictional claims in published maps and institutional affiliations.

\section{Author details}

${ }^{1}$ Department of Nutrition, School of Public Health, Sun Yat-sen University (Northern Campus), Guangzhou 510080, China. ${ }^{2}$ Guangdong Provincial Key Laboratory of Food, Nutrition and Health, Guangzhou 510080, China.

${ }^{3}$ Department of Medical Statistics and Epidemiology, School of Public Health, Sun Yat-sen University (Northern Campus), Guangzhou 510080, China. ${ }^{4}$ Department of Hepatic Surgery,The Third Affiliated Hospital, Sun Yat-sen University, Guangzhou 510630, China. ${ }^{5}$ Department of Cardiology, Sun Yat-sen Memorial Hospital, Sun Yat-sen University, Guangzhou 510120, China.

Received: 25 June 2018 Accepted: 15 October 2018

Published online: 08 November 2018

References

1. Loomba R, Sanyal AJ. The global NAFLD epidemic. Nat Rev Gastroenterol Hepatol. 2013;10:686-90.

2. Younossi ZM, Koenig AB, Abdelatif D, Fazel Y, Henry L, Wymer M. Global epidemiology of nonalcoholic fatty liver disease-meta-analytic assessment of prevalence, incidence, and outcomes. Hepatology. 2016;64:73-84.

3. Li Z, Xue J, Chen P, Chen L, Yan S, Liu L. Prevalence of nonalcoholic fatty liver disease in mainland of China: a meta-analysis of published studies. J Gastroenterol Hepatol. 2014;29:42-51.

4. Marchesini G, Bugianesi E, Forlani G, Cerrelli F, Lenzi M, Manini R, et al. Nonalcoholic fatty liver, steatohepatitis, and the metabolic syndrome. Hepatology. 2003;37:917-23.

5. Jacobs M, van Greevenbroek MM, van der Kallen CJ, Ferreira I, Feskens EJ, Jansen $\mathrm{EH}$, et al. The association between the metabolic syndrome and alanine amino transferase is mediated by insulin resistance via related metabolic intermediates (the cohort on diabetes and atherosclerosis Maastricht [CODAM] study). Metabolism. 2011;60:969-75.

6. Han E, Lee YH. Non-alcoholic fatty liver disease: the emerging burden in Cardiometabolic and renal diseases. Diabetes Metab J. 2017;41:430-7.

7. Brunt EM. Pathology of nonalcoholic fatty liver disease. Nat Rev Gastroenterol Hepatol. 2010;7:195-203.

8. Brunt EM, Wong WW, Nobili V, Day CP, Sookoian S, Maher JJ, et al. Nonalcoholic fatty liver disease. Nat Rev Dis Primers. 2015;1:15080.

9. Hammond GL, Bocchinfuso WP. Sex hormone-binding globulin: gene organization and structure/function analyses. Horm Res. 1996;45:197-201.

10. Joseph DR. Structure, function, and regulation of androgen-binding protein/ sex hormone-binding globulin. Vitam Horm. 1994;49:197-280.

11. Glass AR, Swerdloff RS, Bray GA, Dahms WT, Atkinson RL. Low serum testosterone and sex-hormone-binding-globulin in massively obese men. J. Clin Endocrinol Metab. 1977;45:1211-9.

12. Hammoud A, Gibson M, Hunt SC, Adams TD, Carrell DT, Kolotkin RL, et al. Effect of roux-en-Y gastric bypass surgery on the sex steroids and quality of life in obese men. J Clin Endocrinol Metab. 2009;94:1329-32.

13. Laaksonen DE, Niskanen L, Punnonen K, Nyyssonen K, Tuomainen TP, Salonen $\mathrm{R}$, et al. Sex hormones, inflammation and the metabolic syndrome: a population-based study. Eur J Endocrinol. 2003;149:601-8.

14. Le TN, Nestler JE, Strauss JF 3rd, Wickham EP 3rd. Sex hormone-binding globulin and type 2 diabetes mellitus. Trends Endocrinol Metab. 2012; 23:32-40.

15. Kavanagh K, Espeland MA, Sutton-Tyrrell K, Barinas-Mitchell E, El Khoudary SR, Wildman RP. Liver fat and SHBG affect insulin resistance in midlife 
women: the study of Women's health across the nation (SWAN). Obesity (Silver Spring). 2013;21:1031-8.

16. Daka B, Rosen T, Jansson PA, Rastam L, Larsson CA, Lindblad U. Inverse association between serum insulin and sex hormone-binding globulin in a population survey in Sweden. Endocr Connect. 2013;2:18-22.

17. Shin JY, Kim SK, Lee MY, Kim HS, Ye Bl, Shin YG, et al. Serum sex hormonebinding globulin levels are independently associated with nonalcoholic fatty liver disease in people with type 2 diabetes. Diabetes Res Clin Pract. 2011;94:156-62.

18. Hua $X$, Sun $Y$, Zhong $Y$, Feng $W$, Huang H, Wang W, et al. Low serum sex hormone-binding globulin is associated with nonalcoholic fatty liver disease in type 2 diabetic patients. Clin Endocrinol. 2014;80:877-83.

19. Brzozowska MM, Ostapowicz G, Weltman MD. An association between nonalcoholic fatty liver disease and polycystic ovarian syndrome. J Gastroenterol Hepatol. 2009;24:243-7.

20. Zhang J, Hu J, Zhang C, Jiao Y, Kong X, Wang W. Analyses of risk factors for polycystic ovary syndrome complicated with non-alcoholic fatty liver disease. Exp Ther Med. 2018;15:4259-64.

21. Wang N, Zhai H, Zhu C, Li Q, Han B, Chen Y, et al. Combined Association of Vitamin D and sex Hormone Binding Globulin with Nonalcoholic Fatty Liver Disease in men and postmenopausal women: a cross-sectional study. Medicine (Baltimore). 2016;95:e2621.

22. Tian GX, Sun Y, Pang CJ, Tan AH, Gao Y, Zhang HY, et al. Oestradiol is a protective factor for non-alcoholic fatty liver disease in healthy men. Obes Rev. 2012;13:381-7.

23. Jaruvongvanich V, Sanguankeo A, Riangwiwat T, Upala S. Testosterone, sex hormone-binding globulin and nonalcoholic fatty liver disease: a systematic review and meta-analysis. Ann Hepatol. 2017;16:382-94.

24. Rhee J, Ge H, Yang W, Fan M, Handschin C, Cooper M, et al. Partnership of PGC-1alpha and HNF4alpha in the regulation of lipoprotein metabolism. J Biol Chem. 2006;281:14683-90.

25. Janne M, Hammond GL. Hepatocyte nuclear factor-4 controls transcription from a TATA-less human sex hormone-binding globulin gene promoter. J Biol Chem. 1998;273:34105-14.

26. Selva DM, Hogeveen KN, Innis SM, Hammond GL. Monosaccharide-induced lipogenesis regulates the human hepatic sex hormone-binding globulin gene. J Clin Invest. 2007;117:3979-87.

27. Selva DM, Hammond GL. Thyroid hormones act indirectly to increase sex hormone-binding globulin production by liver via hepatocyte nuclear factor-4alpha. J Mol Endocrinol. 2009;43:19-27.

28. Simo R, Saez-Lopez C, Lecube A, Hernandez C, Fort JM, Selva DM. Adiponectin upregulates SHBG production: molecular mechanisms and potential implications. Endocrinology. 2014;155:2820-30.

29. Fan JG, Jia JD, Li YM, Wang BY, Lu LG, Shi JP, et al. Guidelines for the diagnosis and management of nonalcoholic fatty liver disease: update 2010: (published in Chinese on Chinese journal of hepatology 2010; 18:163-166). J Dig Dis. 2011;12:38-44.

30. Ratziu V, Bellentani S, Cortez-Pinto H, Day C, Marchesini G. A position statement on NAFLD/NASH based on the EASL 2009 special conference. J Hepatol. 2010;53:372-84.

31. Zhou BF. Effect of body mass index on all-cause mortality and incidence of cardiovascular diseases--report for meta-analysis of prospective studies open optimal cut-off points of body mass index in Chinese adults. Biomed Environ Sci. 2002;15:245-52.

32. Ainsworth BE, Haskell WL, Herrmann SD, Meckes N, Bassett DR Jr, TudorLocke C, et al. 2011 compendium of physical activities: a second update of codes and MET values. Med Sci Sports Exerc. 2011;43:1575-81.

33. Liu YH, Xu Y, Wen YB, Guan K, Ling WH, He LP, et al. Association of weightadjusted body fat and fat distribution with bone mineral density in middleaged chinese adults: a cross-sectional study. PLoS One. 2013;8:e63339.

34. Zeng MD, Fan JG, Lu LG, Li YM, Chen CW, Wang BY, et al. Guidelines for the diagnosis and treatment of nonalcoholic fatty liver diseases. J Dig Dis. 2008;9:108-12.

35. Kleiner DE, Brunt EM, Van Natta M, Behling C, Contos MJ, Cummings OW, et al. Design and validation of a histological scoring system for nonalcoholic fatty liver disease. Hepatology. 2005;41:1313-21.

36. Williams CD, Stengel J, Asike MI, Torres DM, Shaw J, Contreras M, et al. Prevalence of nonalcoholic fatty liver disease and nonalcoholic steatohepatitis among a largely middle-aged population utilizing ultrasound and liver biopsy: a prospective study. Gastroenterology. 2011;140:124-31.

37. Jornayvaz FR, Shulman Gl. Diacylglycerol activation of protein kinase Cepsilon and hepatic insulin resistance. Cell Metab. 2012;15:574-84.
38. Simo R, Barbosa-Desongles A, Hernandez C, Selva DM. IL 1 beta downregulation of sex hormone-binding globulin production by decreasing HNF4alpha via MEK-1/2 and JNK MAPK pathways. Mol Endocrinol. 2012;26:1917-27.

39. Simo R, Barbosa-Desongles A, Lecube A, Hernandez C, Selva DM. Potential role of tumor necrosis factor-alpha in downregulating sex hormone-binding globulin. Diabetes. 2012;61:372-82.

40. Simo R, Barbosa-Desongles A, Saez-Lopez C, Lecube A, Hernandez C, Selva DM. Molecular mechanism of TNFalpha-induced Down-regulation of SHBG expression. Mol Endocrinol. 2012;26:438-46.

41. Pugeat M, Nader N, Hogeveen K, Raverot G, Dechaud H, Grenot C. Sex hormone-binding globulin gene expression in the liver: drugs and the metabolic syndrome. Mol Cell Endocrinol. 2010;316:53-9.

42. Ding EL, Song Y, Manson JE, Hunter DJ, Lee CC, Rifai N, et al. Sex hormonebinding globulin and risk of type 2 diabetes in women and men. $N$ Engl J Med. 2009;361:1152-63.

43. Saez-Lopez C, Rivera-Gimenez M, Hernandez C, Simo R, Selva DM. SHBGC57BL/ksJ-db/db: a new mouse model to study SHBG expression and regulation during obesity development. Endocrinology. 2015;156:4571-81.

44. Saez-Lopez C, Barbosa-Desongles A, Hernandez C, Dyer RA, Innis SM, Simo $\mathrm{R}$, et al. Sex hormone-binding globulin reduction in metabolic disorders may play a role in NAFLD development. Endocrinology. 2017;158:545-59.

45. Frith J, Day CP, Henderson E, Burt AD, Newton JL. Non-alcoholic fatty liver disease in older people. Gerontology. 2009;55:607-13.

46. Zhai HL, Wang NJ, Han B, Li Q, Chen Y, Zhu CF, et al. Low vitamin D levels and non-alcoholic fatty liver disease, evidence for their independent association in men in East China: a cross-sectional study (survey on prevalence in East China for metabolic diseases and risk factors (SPECTChina)). Br J Nutr. 2016;115:1352-9.

47. Yan J, Xie W, Ou WN, Zhao H, Wang SY, Wang JH, et al. Epidemiological survey and risk factor analysis of fatty liver disease of adult residents, Beijing. China J Gastroenterol Hepatol. 2013;28:1654-9.

48. Seto WK, Yuen MF. Nonalcoholic fatty liver disease in Asia: emerging perspectives. J Gastroenterol. 2017;52:164-74.

\section{Ready to submit your research? Choose BMC and benefit from:}

- fast, convenient online submission

- thorough peer review by experienced researchers in your field

- rapid publication on acceptance

- support for research data, including large and complex data types

- gold Open Access which fosters wider collaboration and increased citations

- maximum visibility for your research: over $100 \mathrm{M}$ website views per year

At BMC, research is always in progress.

Learn more biomedcentral.com/submissions 